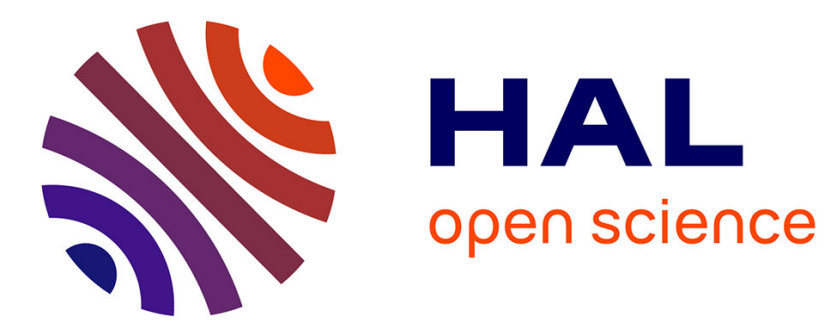

\title{
Les garçons, la mixité et l'animation
}

Sylvie Ayral, Yves Raibaud

\section{To cite this version:}

Sylvie Ayral, Yves Raibaud. Les garçons, la mixité et l'animation. Agora débats/jeunesses, 2009, 51, pp.43-58. 10.3917/agora.051.0043 . halshs-00526008

\section{HAL Id: halshs-00526008 \\ https://shs.hal.science/halshs-00526008}

Submitted on 13 Oct 2010

HAL is a multi-disciplinary open access archive for the deposit and dissemination of scientific research documents, whether they are published or not. The documents may come from teaching and research institutions in France or abroad, or from public or private research centers.
L'archive ouverte pluridisciplinaire HAL, est destinée au dépôt et à la diffusion de documents scientifiques de niveau recherche, publiés ou non, émanant des établissements d'enseignement et de recherche français ou étrangers, des laboratoires publics ou privés. 
Sylvie Ayral est professeure agrégée d'espagnol et prépare une thèse en Sciences de l'Education sur le thème « La fabrique des garçons. Sanction et genre au collège » sous la direction du Professeur Eric Debarbieux de l’Université de Bordeaux 2 Victor Segalen. ayrals@wanadoo.f

Yves Raibaud est géographe, maître de conférences à l'IUT Michel de Montaigne à Bordeaux, membre de l’UMR 5185 ADES. Ses recherches portent sur les rapports entre musique et territoire, genre et loisirs des jeunes et la formation aux métiers de l'animation. Il est responsable pédagogique du master professionnel « Spécialité de l’Ingénierie d’Animation Territoriale » à Bordeaux.

y.raibaud@ades.cnrs.fr

\section{Les garçons, la mixité et l’animation Agora Débats Jeunesse n51, septembre 2009, p. 43 à 58}

Résumé: L'article pose la question des garçons dans un contexte de mixité lorsqu'ils refusent l'autorité, ont des comportements violents et choisissent les activités qui leurs paraissent viriles plutôt que d'autres. L'hypothèse que l'identité masculine est un construit social permet de s'interroger sur la manière dont les garçons se perçoivent et sont perçus. La naturalisation de l'identité masculine masque peut-être un projet pédagogique implicite : faire des garçons de "vrais hommes », ni efféminés, ni homosexuels. Poser le genre comme une variable centrale de la violence sociale permet alors d'inventorier quelques ressources de l'animation permettant d'améliorer les rapports sociaux de sexe.

Les garçons polarisent souvent l'attention et l'énergie des animateurs et des animatrices (ou animateures), rendant difficile la mise en œuvre d'activités mixtes. Ils jouent les « fortes têtes », adoptent des comportements violents et rejettent certaines activités qu'ils estiment dégradantes pour leur condition masculine. L'animation socioculturelle, qui intervient sur le temps libre et ne dispose généralement pas d'un régime de sanctions formelles pour réguler l'indiscipline, a tendance à suivre les garçons sur les terrains favorables à l'expression de la masculinité : sports virils, culture rock, jeux guerriers, cyberespace fantastique et violent. Notre hypothèse est que ce traitement particulier fait aux garçons, loin de favoriser l'approche égalitaire des sexes, participe à une surconstruction de la masculinité. Le virilisme, le sexisme et l'homophobie qui en découlent ne seraient pas les conséquences d'une condition masculine naturelle mais des construits sociaux, encouragés implicitement par la communauté éducative afin de fabriquer des « vrais » garçons.

Dans un premier temps cet article interroge le champ de l'animation en tant que contexte de mixité de sexe ${ }^{1}$. Dans un deuxième temps il invite à analyser le discours des acteurs, recueilli lors d'enquêtes par entretiens en 2007-2008 ${ }^{2}$. Qu'est-ce qui se joue réellement pour les garçons dans leurs conflits avec les adultes qui les encadrent et avec leurs pairs ? Nous verrons comment leur comportement est d'abord distingué, puis accepté pour, en bout de parcours, générer un discours naturalisant («les garçons sont comme ça, c’est les hormones ! »). Il devient alors difficile pour eux d’échapper à une norme masculine qui fait consensus aussi bien chez les filles, habituées à être reléguées et malmenées, que chez les parents et adultes qui redoutent une homosexualité latente ${ }^{3}$. Dans ces conditions il apparaît

\footnotetext{
${ }^{1}$ Voir (entre autres) sur ce sujet les travaux de Judit Vari sur les garçons « difficiles » face à l'autorité féminine, de Elisa Herman sur l'idéologie de la complémentarité des rôles sexués dans l'animation et de Magalie Bacou sur la mixité dans l'animation in Mixité, Parité, Genre dans les métiers de l'animation, s.d. J.C. Gillet et Y. Raibaud, 2006.

${ }^{2}$ L'enquête porte sur des entretiens réalisés avec 15 groupes mixtes de garçons et filles de 12 à 15 ans et 15 adultes qui les encadrent ; elle a été réalisée en 2007 à l'occasion d'un travail de master recherche (Sylvie Ayral, 2007). L’article a mobilisé également un travail d'enquête réalisé auprès de professionnel.le.s de l'animation et des loisirs à l'occasion d'un mémoire professionnel sur les loisirs culturels des jeunes (Marie-Christine Barthaburu, 2007).

${ }^{3}$ Nous ne retenons pas comme catégorie explicative l’adolescence et la puberté dans la construction des identités sexuées, mais nous ne les nions pas non plus dans la mesure où adolescence et puberté font partie des représentations communes à la communauté éducative des adultes et à celle des enfants et des jeunes. Le parti pris consiste à interroger ces représentations, de montrer comment elles sont performatives dans le choix de l'offre de loisirs dans l'animation puis de proposer un cadre de réflexion pour l'analyse des pratiques professionnelles (cf. approche ethnométhodologique, Garfinkel 2007 [1967]; interactionnisme, Goffman 2002 [1977]).
} 
que viriliser les garçons et féminiser les filles pourraient bien être des objectifs pédagogiques implicites, particulièrement dans la mise en œuvre de loisirs culturels et sportifs dans le secteur de l'animation. Dans un troisième temps, l'article propose de passer de l'analyse des représentations à l'analyse des pratiques professionnelles, puis d'examiner brièvement quelques ressources militantes qui ont été développées par les mouvements d'éducation populaire et l'animation socioculturelle sur ces sujets. De nombreuses initiatives et alternatives existent, un peu partout en France et ailleurs, qui ne demandent qu'à être reprises et étendues, afin de "donner un véritable sens à un travail sur l'égalité des sexes dans les conditions classiques de la mixité » (Lang, 2000).

\section{Mixité et genre ${ }^{4}$ à l’école et dans les temps de loisirs}

L’école et les loisirs organisés en périphérie de l'école (animation socioculturelle, pratiques sportives et artistiques etc.) sont les principales instances de prise en charge des jeunes hors de leur famille. Même si le contexte légal a imposé en France la mixité de sexe à l'école, celle-ci est plus ou moins effective dans les temps de vie des enfants et des jeunes d'âge scolaire. A l'échelle d'une journée, la mixité est totale à l'école mais relative dans les temps de récréation, de repas, dans les activités décloisonnées, pendant l’accueil périscolaire et l'offre de loisirs organisés.

Quiconque entre dans un collège, un centre de loisirs ou une maison de quartier constate d'abord que les enfants ont tendance à se regrouper par sexe puis, assez vite, que les garçons sont plus agités, plus bruyants, qu'ils monopolisent l'espace commun (les filles se trouvant reléguées dans les coins ou les espaces périphériques) et qu'ils transgressent davantage les règles. Dans les loisirs organisés à la sortie de l'école on peut se demander si le «temps libre » ne sert pas de temps de "temps de décompression » après l'obligation de mixité vécue pendant la journée de classe où les élèves sont regroupés de façon artificielle et confrontés à chaque instant à l'altérité sexuée. Comme dans la cour de récréation, filles et garçons se séparent pour se coopter dans des activités communes. Les activités proposées se font dans le sens du libre choix, mais l'énoncé même de ces choix (jeux d'extérieur ou activités manuelles, foot ou danse...) les oriente implicitement vers des groupes non mixtes (Bacou, 2006). L' " arrangement des sexes » fonctionne à plein et fixe durablement les garçons et les filles dans des pratiques qui les séparent, ce que l'on retrouve dans les activités volontaires, sportives ou culturelles, que certains choisissent par la suite.

On pourrait supposer qu'à l'école et dans les moments de loisirs, espaces/temps de mixité, la violence des rapports sociaux de sexe s'atténue. En réalité ces espaces/temps apparaissent davantage comme des univers de confrontations intersexes et d'activation de stéréotypes de genre (représentation de soi en tant qu'homme ou en tant que femme) que comme des espaces de coéducation des sexes. La coprésence des filles et des garçons dans le système éducatif et, dans une moindre mesure dans le système d'animation (Augustin, Gillet,

\footnotetext{
${ }^{4}$ Le genre distingue le sexe biologique du sexe social, postule que les caractéristiques associées à chaque sexe sont socialement construites dans une relation d'opposition, appréhende les relations sociales entre les sexes comme un rapport hiérarchisé. Le genre est à l'intersection d'autres rapports de pouvoir comme la classe, l'âge et la race. Il invite à observer les différences non seulement entre hommes et femmes mais également entre les femmes elles-mêmes ou les hommes euxmêmes, en cessant de les considérer comme deux groupes distincts et homogènes. Il questionne les stéréotypes de sexe qui imprègnent les représentations sociales (J. Butler, 1990; L. Bereni et alii, 2008). Le genre s'élabore dans les interactions quotidiennes, il est fait et refait chaque fois qu'une personne sexuée en rencontre une autre ou encore chaque fois qu'un individu essaye de classer l'autre dans un sexe afin de savoir comment se comporter avec lui (E. Goffman, 2002 [1977]). Il y a autant de socialisations de genre qu'il y a d'instances de socialisation ou de sous-espaces sociaux sexués (politique, travail, école, groupe de pairs, sports et loisirs, médias, sexualité, famille, religion etc.). Pourtant, dans presque toutes ces instances, virilisme, sexisme et homophobie apparaissent comme des éléments récurrents de l'identité masculine (D. WelzerLang, 2004).
} 
2000) alimente de nombreux débats. Doit-on l'appeler mixité ? Coéducation ? Quels en sont les effets positifs ? Négatifs ? Parler de mixité consiste souvent à célébrer une valeur drainant avec elle les notions d'égalité, de respect entre les sexes et incarnant la vertu démocratique. Dans les faits la mixité n'entraîne t-elle pas, au contraire, filles et garçons à afficher plus ostensiblement les stéréotypes attachés à leur sexe ? (Fize, 2003). " Ce qui est en jeu, c’est le sens et la place du sexué et/ou du sexuel [...]. Dans la 'mixité ', la dimension sexuelle des garçons et des filles, des hommes et des femmes est l'objet d'une négation pure et simple » (Lelièvre et Lec, 2005, p.73).

Le rapport à l'autre sexe dans le cadre de la mixité scolaire et extrascolaire pose un problème politiquement et pédagogiquement rarement pris en compte. La différence entre « coéducation » et « mixité » n'est donc pas simplement d'ordre terminologique : le terme de « coéducation » implique une finalité éducative.

\section{Injonction sociale à la virilité : convergence des discours}

Pierre Bourdieu dans La domination masculine (1998) décrit la longue socialisation qu'il faut aux hommes pour se démarquer des femmes, depuis les jeux et joutes viriles, jusqu'au fétichisme de la virilité marqué par le désir de dominer les autres hommes et, secondairement, les femmes. L'habitus masculin décrit par P. Bourdieu se traduit par une tension permanente " qu'impose à chaque homme le devoir d'affirmer en toute circonstance sa virilité (...). La virilité, entendue comme capacité reproductive, sexuelle et sociale, mais aussi comme aptitude au combat et à l'exercice de la violence (...). La virilité doit être validée par les autres hommes (...), ceux qui n'appartiennent pas aux vrais hommes vont « perdre la face » et être traités de « mauviettes, de « femmelettes », de « pédés » (id., p.58).

Le fait de perturber l'ordre, le défi, l'insolence, la violence sexiste et / ou homophobe ne relèvent-ils pas, en fin de compte, chez les garçons, d'une parade sexuée masculine (Goffman, 2002 [1977]) pour se démarquer des filles et se faire admettre dans le groupe des hommes ? A travers leurs comportements d'opposition à l'école ou dans les loisirs organisés en périphérie de l'école les garçons semblent vouloir confirmer leur identité masculine, mais ne seraient-ils pas aussi guidés par une injonction sociale qui leur commande de devenir des hommes virils et dominants?

Une quinzaine d'entretiens portant sur ces questions ont été conduits avec des groupes non mixtes de cinq jeunes de 12 à 15 ans de la région de Bordeaux (France) et une autre quinzaine d'entretiens individuels avec les adultes, enseignant.e.s, éducateur.e.s et animateur.e.s qui les encadrent (Ayral, 2007; Barthaburu, 2007). Sans revenir sur la totalité des études, voici quelques extraits qui témoignent des représentations que les personnes interrogées se font des garçons.

\section{Les garçons parlent d'eux-mêmes}

Les garçons parlent d' " un tempérament » masculin qui s'imposerait aux hommes et donc à eux depuis toujours, d’une agressivité explosive et incontrôlable.

« Et nous, on aime la bagarre et pas...pas les filles... non, franchement, de toutes façons, la bagarre, ça a toujours été sur l'humain, bon, souvent, plus, plus sur les garçons...»

«... Ouais, y a le tempérament !»

Q. : Vous avez un tempérament, c'est-à-dire, est-ce que vous pouvez m'expliquer ?

«Y a les filles, par exemple, quand elles ont des problèmes, elles...elles vont parler...même si elles doivent se traiter, ....mais alors que les garçons, non...ça...ça parle trente secondes et après bam ! » (...) « Les garçons, ça tape et après ça parle ». 


\section{Q. : Comment vous l'expliquez ?}

« Ça, c'est dans la nature. C’est la nature de l’homme, voilà. On a été fait comme ça, c'est dans la tête. Et même si on...même si...des fois y en a qui le pensent pas...y a un jour ou un autre, ça...on accumule, on explose ...voilà ».

« J'sais pas mais...l'exemple sur les guerres...ça va depuis, depuis le début...enfin...y a toujours eu, y a toujours eu des guerres dans le monde et c'était, c'était jamais des guerres de femmes, c'était toujours des hommes qui se battaient ».

«Y en a déjà eu des femmes, bon, mais voilà...c'était pour s'occuper...c'étaient les infirmières ".

Un garçon régulièrement sanctionné au collège explique ce qu'il ressent lorsqu'il est puni (ce qu’il recherche, manifestement). Il dit éprouver une sensation physique agréable, une « montée d'adrénaline » excitante.

Q. : Quel effet produit une sanction grave ou une exclusion sur l'élève puni ?

« Franchement, rien du tout. Parce que au contraire, quand un élève est...est exclu, moi, je peux vous le dire parce que je suis exclu bientôt trois jours, mais justement, je suis content de partir... Moi, enfin...c'est... c'est vrai que d'avoir des punitions ou d'être renvoyé, c'est...pour moi c'est quelque chose aussi de bien, parce que bon, c'est...ça, ça donne des adrénalines et ça donne des pulsations au cœur et on sait pas ce qui nous attend, donc, ça nous fait quelque chose vraiment de fort quoi, c'est... c'est...

Q. : C'est excitant?

« Voilà. C’est...c'est justement, c'est ça qui, que je cherche moi, quand je me fais punir, quoi ».

\section{b) Les filles parlent des garçons}

Les filles évoquent leur force physique moindre: seuls les garçons auraient les capacités requises pour se battre...Sur cet aspect, elles ne se sentent pas « à la hauteur ».

«Ils sont plus forts que les filles, c'est... euh...enfin nous on est des nulles, quoi. Enfin, pour eux, c'est...c'est vrai ils sont plus excités que nous, ils se parlent entre eux, ils sont les plus forts...euh...ils veulent montrer qu'ils nous...qu'ils nous dominent». «Euh... aussi les garçons euh...eh ben, pour régler les problèmes, ils se battent, tandis que les filles, je pense que pour régler les problèmes, elles parlent plutôt entre elles, elles essaient d'expliquer les choses ».

Q. : Est-ce que la violence des garçons s'exerce sur les autres garçons ou sur les filles? " Oui...ils disent que ça se fait pas de taper une fille. Enfin, pas entre nous ».

Q. : Pourquoi vous pensez que ça ne se fait pas de taper les filles chez les garçons ? « Parce qu'il a le pouvoir donc il va vouloir montrer qu'il est plus fort que les autres garçons».

Elles semblent tout à fait conscientes d'une forme de domination, mais ne la remettent pas en question. Il s'agit d'enjeux de pouvoir entre garçons: si un garçon est plus fort que les autres garçons, il l'est encore plus que toutes les filles

« Et aussi parce que...enfin...les filles elles ont pas, elles sont pas pareilles, enfin... ça dépend je sais mais, souvent les garçons, ils sont plus forts que les filles...

Q. : Physiquement, tu veux dire ?

- Oui. Ils se battent plus, tandis que les filles, elles se battent moins que les garçons.

Q. : Alors vous me dites que oui, souvent ils font exprès de se faire remarquer et dans quel but d'après vous ?

- De se sentir intéressants. 
- Pour montrer leur force et euh...voilà ».

c) Les adultes parlent des garçons : mobilisation de savoirs « scientifiques »

Les enseignant.e.s et animateur.e.s consulté.e.s en entretien évoquent des comportements très différents d'un sexe à l'autre La première explication est cherchée dans la nature et s'appuie sur la biologie.

«L'instinct masculin refait toujours surface » $(\mathrm{H})^{5}$. «Les gènes ? » $(\mathrm{H})$. « Peut-être est-ce une question de gènes ou d'hormones » $(\mathrm{F})$. « Les garçons sont naturellement plus turbulents que les filles. Pour avoir élevé des quantités d'animaux, j’ai pu observer que les mâles sont naturellement plus agressifs. Quand on veut un animal doux on choisit une femelle» (F).

«Des études scientifiques prouvent que l'apport d'hormones mâles augmente l'agressivité [...] L'agressivité des garçons est naturelle, mais elle peut être soit encouragée par une éducation machiste, soit corrigée » (F).

Hormones mâles et hormones femelles sont une « loi de nature ». En faisant référence à la zoologie on argue du côté « animal » de cette différence pour justifier son côté immuable. Mais, les hormones, ça ne se voit pas : il est donc important de faire apparaître cette nature sous son aspect le plus visible.

« Peut-être est-ce en relation avec la puberté ? [...] Chez les garçons la puberté est exogène, la transformation de l'appareil génital est extérieure, elle est doublée d'un développement musculaire ».

« ... chez les jeunes filles, la puberté est à l’intérieur... appareil génital intérieur...le développement est plus intérieur, plus intellectualisé » $(\mathrm{H})$

La naturalisation s'appuie sur la différence « incontestable » de l'aspect des organes génitaux : d'un côté un pénis extérieur, visible, démonstratif ; de l'autre un vagin invisible, une «absence de sexe». La puberté adolescente se manifesterait ainsi par le surinvestissement de ces différences visibles, qui participent à indiquer clairement les rôles sexués.

«Euh, moi, je commencerais par dire que les garçons ont une forme de violence non contenue qui a besoin de déborder, et euh...oui la puberté des garçons est plus extériorisée » $(\mathrm{H})$

«...ça se manifeste par un développement physique, un développement sexuel, comment dirai-je, évident, apparent, à mon sens ça entraîne une forme de compétition entre les garçons ... cette compétition liée aux transformations de la puberté, elle se manifeste dans leur relations, dans le physique, à l'inverse, les...les transformations féminines me semblent beaucoup plus intérieures.. plus raisonnées, euh...les violences chez les filles sont plus psychologiques et personnelles » $(\mathrm{H})$

«Les garçons, on sent de suite qu'il y a une forme...chez les filles c'est beaucoup plus intérieur » $(\mathrm{H})$

Cette puberté des garçons qui fait grossir leur pénis et développe leurs muscles les inciterait à la comparaison, à la compétition. Les filles, au contraire, se développeraient «à l'intérieur ", on pourrait presque dire "en secret", ce qui les amènerait à une vie psychologique plus intense mais dangereuse...pour elles.

\footnotetext{
${ }^{5} \mathrm{H}=$ homme. $\mathrm{F}=$ Femme
} 
« C’est un problème génétique [...]. Ils sont plus agressifs que les filles, ils font la guerre, hein ? ». « ...Le garçon est plus agressif que la fille » $(\mathrm{H})$

«La fille, génétiquement ou par, par sa physiologie, me paraît plus calme, euh... plus apaisante qu'un garçon. La violence des filles se retourne contre elles.» (F)

Les explications glissent vers la différence culturelle. Depuis des millénaires se seraient créées une « culture masculine » et une « culture féminine » qui se perpétuent et sont indéracinables. Le masculin serait lié depuis « la nuit des temps » à la guerre et à la violence. Que les garçons se confrontent sur un terrain de foot plutôt que dans des guerres serait donc finalement un moindre mal...

Ce glissement vers une différence culturelle vaut particulièrement lorsqu'il s'agit de populations issues de l'immigration. A la nature de l'homme viennent se superposer les supposées «cultures ethniques », pensées comme des cultures archaïques. Les adultes qui encadrent les garçons ne présentent plus alors la nature masculine comme une affaire hormonale, mais comme un choc de civilisation.

« Ici, le problème des garçons, c'est surtout les gens du voyage... Chez, les gitans, les hommes sont les rois... Il ne faut pas toucher à un garçon gitan, sinon, on a toute la famille sur le dos » $(\mathrm{F})$

« Le problème, à C., c'est les garçons maghrébins... La culture musulmane, c'est les hommes qui dominent ... Les grands frères surveillent leurs sœurs » $(\mathrm{F})$

« Dans les familles maghrébines en particulier, l'homme dirige tout, c'est à lui qu'il faut s'adresser pour les autorisations de sortie des enfants » $(\mathrm{H})$

d) La sexualité incertaine des « adolescent.e.s » : les protéger du risque homosexuel.

Non seulement la naturalisation des rôles sociaux de sexe homogénéise les attitudes de tous les garçons d'un côté et de toutes les filles de l'autre mais elle est la source d'une « police du genre » qui surveille toute possibilité de déviation de la norme :

« Dès la fin de l'école primaire, un garçon qui chante ou qui danse se fait traiter de «gonzesse » ou de "pédé » [...]. Les jeux sont codifiés, et surveillés par les garçons les plus virils qui portent des jugements et agressent les garçons qui jouent avec les filles [...]. Le sport spontané (foot) devient l’activité principale des garçons, assez violente ${ }^{6}$.

La peur de faire des garçons efféminés guide les parents qui les inscrivent dans des activités en fonction des attentes sexuées présumées de la société, surtout si leur garçon présente quelques traits un peu délicats. Ainsi cette maman dont le garçon arrête la musique et qui l'inscrit au judo :

"A l'école, il se fait battre par les autres garçons qui le traitent de fille [...]. On aimerait qu'il apprenne à se défendre » ${ }^{7}$.

La croyance que l'adolescence est un âge indécis sur le plan de la sexualité renforce la crainte que plus tard, l'enfant ne «bascule» dans l'homosexualité, risque pour sa santé psychique et physique (le sida).

\footnotetext{
${ }^{6}$ Extraits d'entretiens, in Barthaburu (2007)

${ }^{7}$ Barthaburu, id.
} 
« Je n’ai pas voulu inscrire ma fille au hand-ball car il paraît que c’est un sport où il $\mathrm{y}$ a beaucoup de lesbiennes ».

« La danse pour Baptiste ? non, vraiment... Je veux pas... j’aurais un peu peur qu'il devienne... heu... comment dire... homosexuel, quoi.... ${ }^{8}$.

Pour éviter ce risque, et faute de pouvoir agir sur le plan génétique ou hormonal, il faudrait donc éloigner les garçons et les filles des lieux et activités supposés être fréquentés par les homosexuel.le.s, les aider à trouver la sexualité la plus normale possible en leur évitant tout risque de «tentation » ou de " contamination ». Dans ces conditions l'attitude sexiste et homophobe que peut exprimer ouvertement un garçon rassure car elle démontre son hétérosexualité.

\section{Des représentations vers l'analyse des pratiques professionnelles}

Analyser ces représentations permet de mieux comprendre des relations garçons filles qui, au dire des animateur.e.s, auraient tendance à se détériorer: violences sexistes et homophobes, notamment verbales, seraient en augmentation ${ }^{9}$. Le fait d'observer comment ces représentations agissent dans les pratiques professionnelles et en particulier dans le choix de l'offre de loisirs peut alors permettre d'engager un travail réflexif.

Que penser par exemple de l'argument qui évoque la nécessité de privilégier un « entre soi » des garçons et des filles au moment où se construit leur identité ? Les entretiens nous montrent le risque qu'il y aurait d'aligner ainsi l'identité des garçons sur le modèle du "plus fort et du plus viril » et celui des filles sur le modèle de «la plus docile et la plus gracieuse », et la difficulté supplémentaire que cela représenterait pour les garçons et les filles qui ne correspondent pas aux critères médiatisés de la masculinité ou de la féminité.

Cela peut avoir comme conséquence d'appauvrir les possibilités des un.e.s et des autres en terme de pratiques sociales: garçons maladroits dans la danse, le chant ou les activités artistiques, filles peu sportives et mal à l'aise dans les espaces où il est nécessaire de conquérir physiquement sa place (ce qu'illustrent de nombreux travaux qui montrent, au-delà des stéréotypes, les processus qui construisent au jour le jour ces multiples et minuscules ségrégation ${ }^{10}$ ). Mais on peut aussi invoquer l'argument contraire: certains travaux scientifiques évoquent le fait que dans les activités mixtes s'opère spontanément un partage des tâches assignant à chaque sexe des rôles prédéfinis, quel que soit l'âge des enfants ; il faudrait donc rompre cet "arrangement des sexes » pour permettre une redistribution des tâches entre garçons d'un côté, filles de l'autre.

La notion de mixité active, dans l'esprit des pédagogies nouvelles, consisterait dans tous les cas à discuter les rôles assignés à chacun en montrant l'arbitraire des situations concrètes dans lesquelles se répartissent généralement les rôles sexués. Outre l'aspect ludique et stimulant de la discussion, chercher les situations inversées peut permettre la mise en valeur du « milieu » du groupe mixte (par exemple les garçons sensibles et les filles décidées) plutôt que les polarités extrêmes du stéréotype de genre (les garçons hypervirils, les filles ultraféminines).

\footnotetext{
${ }^{8}$ Barthaburu, ibid.

${ }^{9}$ Guillemet J.P. et Masson Cl. (2008), Le sexisme dans les territoires de Bastide-Benauge et de Saint-Michel à Bordeaux, diagnostic réalisé pour le Conseil Local de Sécurité et de Prévention de la Délinquance (CLSPD) de la ville de Bordeaux, Université de Bordeaux 2 Victor Segalen.

${ }^{10}$ Par exemple Vigneron (2006), «Les inégalités de réussite en EPS entre filles et garçons : déterminisme biologique ou fabrication scolaire ? ", Revue française de pédagogie, n¹54, p.19-29.
} 
Posons comme constat que le sport est une activité plus favorable aux hommes qu'aux femmes même s’il existe des pratiques sportives et des compétitions féminines ${ }^{11}$. Le sport ne consiste t-il pas à restaurer le mythe de la force physique (dans un monde où celle-ci est moins valorisée qu'autrefois par le «travail de force») donc, au final, la suprématie de l'homme sur la femme ? Dans ces conditions, quelle place peut-il y avoir pour le sport mixte? Certaines expériences de rugby mixte «au pied des tours » ${ }^{12}$ montrent des expériences réussies de mixité sociale, ethnique et de genre. Les règles du jeu de ce sport collectif de combat deviennent les bases d'une éducation à la citoyenneté : respect des règles, respect de l'autre, esprit collectif. Des sports alternatifs comme "l'ultimate freesbee " ${ }^{13}$ sont par définition mixtes, autoarbitrés, et favorisent le vis-à-vis filles-filles et garçons-garçons lorsque cela est possible sur les bases du fair-play. Le sport mixte peut être une situation qui permet d'interroger les relations intersexes : pourquoi les garçons ne passent-ils pas le ballon à une fille démarquée ? Pourquoi une fille n'a-t-elle pas tenté sa chance en allant seule vers le but ? Ce projet prend réellement tout son sens lorsque le projet éducatif s’inscrit dans la perspective de la formation d'adultes sportif.ve.s « tout au long de leur vie ». Pour un sportif de soixante ans que reste-t-il de la performance acquise à vingt ans dans un climat d'entraînements intensifs, de matchs violents et engagés, de défis physiques ?... une nostalgie des performances passées et de multiples petites séquelles et traumatismes qui forment la pathologie principale des sportifs d'un certain âge. A l'inverse certaines activités sportives de loisirs (volley, badminton, cyclotourisme, randonnée) redeviennent « naturellement » des pratiques mixtes. Encore faut-il qu'hommes et femmes soient capables de « vivre ensemble » dans ce type d'activité : tel pourrait être l'un des enjeux, simple mais majeur, de l'animation socioculturelle lorsqu'elle utilise le sport comme support.

On aurait pu mentionner le cas de la danse « classique » ou « jazz » (ou des disciplines sportives proches telles que la gymnastique rythmique et sportive) pour vérifier que s'il existe des sports uniquement féminins, ceux-ci mettent en valeur la grâce et la représentation normative, sexuelle, du corps féminin (Barthaburu, 2007). Au contraire du principe d'égalité qui institue des compétitions féminines et masculines distinctes dans l'athlétisme par exemple, ces disciplines mettent en avant une performance féminine inscrite au cœur de la représentation masculine du corps de la femme (Bourdieu, 1998). La danse, contrepoint des sports les plus traditionnellement masculins, participe donc de cette division des représentations basée sur le sexisme et l'homophobie. Premièrement le sexisme car on peut imaginer que la performance première des danseuses est la sculpture d'un corps fantasmé par les hommes et sa mise en spectacle non pas dans le jeu d'un " entre soi » féminin mais bien dans celui du regard des hommes, ou bien des femmes telles qu'elles s'imaginent plaire aux hommes (mais pourquoi pas ?) (id.). La femme athlète, musclée et forte n'a pas sa place dans ce spectacle. Deuxièmement l'homophobie car sur tout homme danseur "classique » ou « jazz » plane le soupçon (dans la civilisation occidentale) d'être homosexuel, sauf s'il

\footnotetext{
${ }^{11} 70 \%$ des pratiques sportives en France sont masculines, ce qu’il faut corréler avec les moyens donnés par l'Etat au sport de compétition, avec le salaire des sportifs comparés à celui des sportives etc. Voir sur ce sujet les études sur les pratiques sportives des jeunes filles et des femmes réalisée par le Ministère de l’Egalité et de la Parité Professionnelle (site du MSJSVA) .

${ }^{12}$ C'est-à-dire dans les cités d'habitat social, exemple de l'association Drop de béton dans la périphérie de Bordeaux.

${ }^{13}$ Jeu de position comparable au rugby mais qui utilise le freesbee (assiette plate en plastique conçue pour voler) à la place du ballon.
} 
pratique des danses à caractère « ethnique » dans lesquelles la masculinité s’affirme de façon forte ${ }^{14}$.

Il en est de même pour le chant : les jeunes garçons refusent souvent de chanter car cela révèle que leur voix (avant la mue) est aussi aiguë que celle des filles. Là encore ce n'est pas tant la crainte d'être confondu aux voix féminines qui s'exprime que celle d'être moqué par des camarades virils qui ont décidé que le chant est une activité de filles (Barthaburu, 2007). Faire danser et chanter les garçons est une mission délicate mais prometteuse s’ils en viennent à considérer qu'ils n’en sortiront pas humiliés ni affaiblis mais au contraire épanouis. Il existe de nombreuses solutions pour éviter le "procès » de féminité qui peut être fait par ses pairs à un garçon danseur ou chanteur : pour les danseurs la danse hip-hop et ses battles, la capoeira, les danses traditionnelles collectives ; pour les chanteurs, la chanson rock ou variété, la chorale gospel... Ces variations sont autant de possibilités qui permettent de valoriser l'expression, la sensibilité et le goût des garçons sans leur faire perdre la face.

Les mots pour le dire

Les «situations d'animation » décrites ci-dessus interagissent avec la connaissance intime que les garçons se font jour après jour de leur identité sexuée et de la manière dont elle est en jeu dans leurs pratiques sociales et dans leurs rapports aux autres. Ces rapports s'expriment aussi avec les mots du sexe : «salope, pouffe, t'es bonne » pour s'adresser aux filles, « pédé, enculé, tapette » pour parler aux garçons (Ayral, 2009) ${ }^{15}$. Pourquoi croire que ce langage n'est que de la vulgarité, alors que les garçons interrogés savent tous expliquer précisément ce dont il s'agit ? Ce vocabulaire participe à la connaissance et à la définition des rapports de sexe : lorsqu'un garçon traite un autre d' "enculé », si celui-ci ne répond pas immédiatement par des coups à l'insulte, c'est «qu'il en est un » et il sera de plus en plus sévèrement harcelé et châtié par le groupe de pairs. Quant aux filles qui se font «traiter » lorsqu'elles traversent le quartier où les garçons imposent leur présence hégémonique, il leur faudra probablement masquer ou dissimuler toute trace de féminité si elles ne veulent pas voir redoubler les insultes sexistes (Welzer-Lang, 2004).

La sanction ou une intolérance trop grande au langage «cru » risquant de rompre le contact avec des jeunes inscrits dans des activités volontaires, pourquoi ne pas parler du sexe dans les situations d'animation, à partir des mots qui sont utilisés par les garçons et les filles dans leur vie quotidienne ? Oui, le sexe et l'activité sexuelle, c'est important; oui c'est compliqué les relations sexuelles mais pas tant que ça si cela se fait entre personnes qui sont d'accord; non la taille et la forme du pénis n'ont pas d'importance, pas plus que la largeur et la profondeur du vagin; oui l'homosexualité existe, chez les hommes et chez les femmes, mais aussi la bisexualité, la transexualité... Oui il existe des femmes qui font du sexe leur métier, et des hommes aussi, moins nombreux... Dans ces discussions les animatrices et les filles peuvent être parfois plus à l'aise car parler du corps c'est un peu comme parler du soin, de soi et de la santé. Il se peut au contraire que les garçons aient du sexe une vision performante, ludique et violente qui se prête moins à une conversation posée. La présence d'un animateur pour parler du sexe avec les garçons peut être une bonne transition pour aborder plus tard le sujet « tous ensembles » : voilà un cas des où l'éducation non mixte peut avoir un intérêt pour dénouer des tensions et des frayeurs propres à chaque groupe.

\footnotetext{
${ }^{14}$ L'exception des danses africaines ou ethniques (voire de la danse hip-hop) ne nous dit pas autre chose : si l'on n'est pas homosexuel quand on est un homme qui danse, il est valorisant d'avoir la peau foncée ce qui permet d'accepter que sa sexualité, rendue apparente par la danse, soit attribuée à son origine ethnique et non à son orientation sexuelle.

${ }^{15}$ Enquête sur les insultes à caractère sexiste ou homophobes réalisé auprès de jeunes de 12 à 15 ans dans le cadre d'une thèse de $3^{\circ}$ cycle en sciences de l'éducation (en cours, Ayral, 2009)
} 


\section{Conclusion}

Pour contourner les nombreuses interprétations qui naturalisent les comportements des garçons, et notamment leur sexualité, il nous paraît donc important d'interroger d'abord les représentations que ceux-ci ont d'eux-mêmes et de les confronter aux représentations qu'en ont les filles et les animateur.e.s. C'est ensuite qu'il sera possible de faire une analyse " genrée » des activités qui leur sont proposées à partir des problèmes posés par la mixité, par les compétences sexuées des animateur.e.s ou par les questions de sexualité et de violence dans les rapports sociaux de sexe. Ce type d'analyse de pratiques, si elle est menée de façon collective peut être à notre avis un facteur de changement rapide dans l'amélioration des rapports entre garçons et filles.

Il ne nous paraît pas anodin de centrer ce travail sur les garçons et les animateurs (ce qui n’a pas été fait, à notre connaissance), afin de les aider (nous aider ${ }^{16}$ ) à «... clore le chapitre de la domination masculine (...) Faire l'inventaire de la virilité. Savoir ce que l'on doit en garder. Ce qui est important que les femmes apprennent à son sujet. Et ce qui doit être jeté aux oubliettes » (Welzer-Lang, 2009, 4 de couverture). Gérer les garçons « difficiles » par la tolérance zéro et la répression n’est pas une bonne solution. Prétendre canaliser leur violence naturelle dans des pratiques sportives et culturelles masculines arrive probablement au même résultat, celui qui participe à la reproduction d'une société hétéronormative (et donc sexiste et homophobe), dominée par les « Grands Hommes » (Godelier, 1988). De l'autre côté le chemin est certes plus long, car il suppose de penser au préalable la question de ces garçons difficiles comme une conséquence d'un système global d'éducation. Le fait de poser l’hypothèse (peut-être exagérée ?) qui ferait du genre une variable centrale de la violence sociale nous fait redécouvrir que de nombreuses alternatives existent déjà, que ce soit sous la forme d'expériences accumulées par les mouvements d'éducation populaire, les associations de défense des victimes et des minorités, les acteurs de l'animation et de l'intervention sociale ou le monde syndical et politique.

Augustin J.P., Gillet J.C., L'animation professionnelle, Paris, L’Harmattan, 2000.

Ayral S., La fabrique des garçons. Sanctions et genre dans un collège rural de Gironde, mémoire master 2 s.d. E. Debarbieux, Université de Bordeaux 2 Victor Segalen, 2007.

Bacou M., Genre et animation dans les centres de loisirs, in Mixité, Parité, genre et métiers de l'animation, sd J.C. Gillet et Y. Raibaud, Paris, L’Harmattan, 2006, p. 73 à 86.

Barthaburu M.C . Genre et éducation musicale, mémoire DUMI, Université de Toulouse, 2007.

Bourdieu, P., La domination masculine, Paris Ed. de Minuit, 1998

Butler J., Gender Trouble : feminism and subversion, Paris, La découverte, 1990.

Fize M., Les pièges de la mixité scolaire, Paris, Seuil, 2003.

Garfinkel H., Recherches en ethnométhodologie, Paris, PUF, (2007 [1967])

Gillet J.C, Raibaud Y., Mixité, parité, genre et métiers de l'animation, Paris, L’Harmattan, 2006

Godelier M., Trahir le secret des hommes, Le Genre Humain n 17, 1988.

Goffman E., L'arrangement des sexes, Paris, La Dispute, (2002 [1977]),

Herman E., Le genre en centre de loisirs : faire avec et "mettre de soi », in Mixité, Parité, genre et métiers de l'animation, id. 2006., p. 87 à 101.

LelièvreC., Lec F., Les profs, l'école et la sexualité, Paris, Odile Jacob, 2005.

Octobre S., Les loisirs culturels des 6-14 ans, La Documentation Française, 2004.

Raibaud Y., Des lieux construits par le genre, in revue Géographie et Culture n54, 2005, p.53 à 70.

Raibaud Y., Genre et loisirs des jeunes, in Des femmes et des hommes : un enjeu pour le social ? Empan $n^{\circ} 65$, 2007, p. 67 à 73.

Raibaud Y., Masculinité et espaces publics, l'offensive des cultures urbaines, in Utopies féministes et expérimentations urbaines, s.d. S. Denèfle, P.U.R, Rennes, 2008, p. 141 à 152.

Vari J., Les animatrice face aux garçons «difficiles » : un impensé, l’autorité féminine. in Mixité, Parité, genre et métiers de l'animation, id, 2006, p. 103 à 113.

\footnotetext{
${ }^{16}$ Note d'un des auteur.e.s.
} 
Vigneron C., « Les inégalités de réussite en EPS entre filles et garçons : déterminisme biologique ou fabrication scolaire ? ", Revue française de pédagogie, n¹54, 2006, p.19 à 29.

Welzer-Lang D., Les hommes aussi changent, Paris, Payot, 2004.

Welzer-Lang D, Nous, les mecs, Paris, Payot, 2009. 